\title{
Singular Reduction of Generalized Complex Manifolds
}

Timothy E. GOLDBERG

Donald and Helen Schort School of Mathematics and Computing Sciences, Lenoir-Rhyne University, Hickory, North Carolina 28601, USA

E-mail: timothy.goldberg@lr.edu

URL: http://mat.lr.edu/faculty/goldberg

Received March 24, 2010, in final form October 06, 2010; Published online October 09, 2010

doi:10.3842/SIGMA.2010.081

\begin{abstract}
In this paper, we develop results in the direction of an analogue of Sjamaar and Lerman's singular reduction of Hamiltonian symplectic manifolds in the context of reduction of Hamiltonian generalized complex manifolds (in the sense of Lin and Tolman). Specifically, we prove that if a compact Lie group acts on a generalized complex manifold in a Hamiltonian fashion, then the partition of the global quotient by orbit types induces a partition of the Lin-Tolman quotient into generalized complex manifolds. This result holds also for reduction of Hamiltonian generalized Kähler manifolds.
\end{abstract}

Key words: generalized complex manifold; Hamiltonian action; generalized complex quotient; Lin-Tolman quotient; singular reduction

2010 Mathematics Subject Classification: 53D20; 53D18; 53C15

\section{Introduction}

Generalized complex geometry was introduced by Hitchin in [11], and further developed by his student Gualtieri in his doctoral thesis [9]. It serves as a common ground in which symplectic, Poisson, and complex geometry can meet. For this reason, there has been much effort to import ideas and techniques from these other fields into the generalized complex setting. In particular, many constructions and results from equivariant symplectic geometry have found useful analogues here. One example is that of Hamiltonian group actions and moment maps, developed in [14]. (Similar constructions were developed and examined by other groups, such as $[4,20]$ and [12], but this paper expands specifically on the work of Lin and Tolman.)

Lin and Tolman's construction generalizes the usual symplectic definition, and they go on to prove that one can reduce a generalized complex manifold by its Hamiltonian symmetries, in perfect parallel to Marsden-Weinstein symplectic reduction [15] (sometimes also credited to Meyer [16]). Just as in the symplectic case, in order to ensure that the generalized reduced space is a manifold, one must make an assumption regarding freeness of the group action.

In [19], Lerman and Sjamaar proved that if the symplectic reduced space is not a manifold, then the orbit type stratification of the original symplectic manifold induces the structure of a stratified space (see Definition 1.7 of that paper) on the reduced space, each stratum of which is naturally a symplectic manifold. The main result of this paper, Theorem 5.3, is a first step in the direction of an analagous result for the case of a singular generalized complex reduced space. It states that the singular generalized complex reduced space can be partitioned into disjoint generalized complex manifolds. It is not yet known whether the reduced space in this situation is actually a stratified space. A similar, although distinct, situation was studied in [13], in which the authors considered the singular reduction of Dirac manifolds. They analyzed the global quotient of a Dirac manifold by a proper group action as a differential space, as in [6], and obtained conditions that guarantee the Dirac structure will descend to the quotient space. 
An interesting difference between the symplectic and generalized complex situations is that the generic result of symplectic reduction is a space with at worst orbifold singularities, whereas for the reduction of a twisted generalized complex manifold, the generic result may be a space with worse-than-orbifold singularities. See Remark 5.1 below.

Section 2 is a rapid introduction to some essential notions from generalized complex geometry. Section 3 reviews some important facts about equivariant cohomology and the orbit type stratification of $G$-spaces. Section 4 consists of a summary of Hamiltonian actions and reduction in generalized complex geometry. Finally, Section 5 contains the full statement and proof of our main theorem.

An earlier version of this work appeared in the author's doctoral thesis [8], where many definitions and calculations are explained in great detail.

Throughout, we use the abbreviations "GC" for "generalized complex" and "GK" for "generalized Kähler". Also, we typically use the same notation and nomenclature to refer to both a map and its complex linear extension. Finally, we make use of the musical notation for the map between a vector space and its dual induced by a bilinear form. If $B: V \times V \rightarrow \mathbb{R}$ is a bilinear form on a real vector space $V$, then we will denote by $B^{b}: V \rightarrow V^{*}$ the map $v \mapsto \iota_{v} B:=B(v, \cdot)$, where $\iota_{v}$ denotes the interior product by $v$. If $B$ is non-degenerate, then $B^{b}$ is invertible and we denote its inverse by $B^{\sharp}:=\left(B^{b}\right)^{-1}$. We also use the musical notation for vector bundles, sections of their second symmetric powers, and the associated bundle maps.

\section{Generalized complex geometry}

We begin by giving several standard definitions and results from generalized complex geometry, which can be found in [9] or [3].

For any smooth manifold $M$, the Pontryagin bundle, or generalized tangent bundle, of $M$ is $\mathbb{T} M:=T M \oplus T^{*} M$. This vector bundle carries a natural non-degenerate symmetric metric $\langle\langle\cdot, \cdot\rangle\rangle$ of signature $(n, n)$, defined by

$$
\langle\langle u+\alpha, v+\beta\rangle\rangle:=\frac{1}{2}(\alpha(v)+\beta(u))
$$

for all $x \in M$ and $u+\alpha, v+\beta \in \mathbb{T}_{x} M$. We will use the same notation for the complex bilinear extension of this metric to the complexification $\mathbb{T}_{\mathbb{C}} M:=\mathbb{T} M \otimes_{\mathbb{R}} \mathbb{C}$. These metrics will henceforth be referred to as the standard metrics on $\mathbb{T} M$ and $\mathbb{T}_{\mathbb{C}} M$.

Proposition 2.1. Let $M$ be a manifold. There is a natural bijective correspondence between the following two structures.

1. Complex linear subbundles $E \subset \mathbb{T}_{\mathbb{C}} M$ over $M$ such that $E \cap \bar{E}=0$ and $E$ is maximally isotropic with respect to the standard metric on $\mathbb{T}_{\mathbb{C}} V$. (Here 0 denotes the image of the zero section of $\mathbb{T}_{\mathbb{C}} M \rightarrow M$, as is customary.)

2. Bundle automorphisms $\mathcal{J}$ of $\mathbb{T} M$ over the identity $M \rightarrow M$ such that $\mathcal{J}^{2}=-\mathrm{id}$ and $\mathcal{J}$ is orthogonal with respect to the standard metric on $\mathbb{T} M$.

Definition 2.2. Let $M$ be a manifold. Either of the equivalent structures described in Proposition 2.1 will be called an almost $\boldsymbol{G C}$ structure on $M$. If $M$ is equipped with an almost GC structure $\mathcal{J}$, then $(M, \mathcal{J})$ is an almost $\boldsymbol{G C}$ manifold.

Let $E \subset \mathbb{T}_{\mathbb{C}} M$ be an almost GC structure on $M$, and for each $x \in M$ let $\pi_{x}: \mathbb{T}_{\mathbb{C}, x} M \rightarrow T_{\mathbb{C}, x} M$ be the projection. The type of this almost GC structure at the point $x \in M$ is the complex codimension of $\pi_{x}\left(E_{x}\right)$ in $T_{\mathbb{C}, x} M$ :

$$
\operatorname{type}(E)_{x}=\operatorname{dim}_{\mathbb{C}} T_{\mathbb{C}, x} M-\operatorname{dim}_{\mathbb{C}} \pi_{x}\left(E_{x}\right) .
$$


Let $\mathcal{J}_{1}$ and $\mathcal{J}_{2}$ be commuting almost GC structures on $M$. Then $G:=-\mathcal{J}_{1} \circ \mathcal{J}_{2}$ is an orthogonal and involutive bundle map $\mathbb{T} M \rightarrow \mathbb{T} M$, and there is an associated bilinear form defined by

$$
(\mathcal{X}, \mathcal{Y}) \mapsto\langle\langle G(\mathcal{X}), \mathcal{Y}\rangle\rangle
$$

for all $\mathcal{X}, \mathcal{Y} \in \mathbb{T} M$ in a common fiber. We call $G$ positive definite if its associated bilinear form is positive definite, i.e. if $\langle\langle G(\mathcal{X}), \mathcal{X}\rangle\rangle>0$ for all nonzero $\mathcal{X} \in \mathbb{T} M$. An almost GK structure on $M$ is a pair of commuting almost GC structures $\left(\mathcal{J}_{1}, \mathcal{J}_{2}\right)$ on $M$ such that $G:=-\mathcal{J}_{1} \circ \mathcal{J}_{2}$ is positive definite.

Remark 2.3. Let $M$ be a manifold. A maximally isotropic linear subbundle of $\mathbb{T} M$, respectively $\mathbb{T}_{\mathbb{C}} M$, is called a Dirac structure, respectively complex Dirac structure on $M$. Thus an almost GC structure on $M$ is a complex Dirac structure $E \subset \mathbb{T}_{\mathbb{C}} M$ satisfying $E \cap \bar{E}=\{0\}$.

Definition 2.4. Let $M$ be a manifold, and let $B \in \Omega^{2}(M)$, where $\Omega^{2}(M)$ denotes the space of differential two-forms on $M$. The $B$-transform of $\mathbb{T} M$ defined by $B$ is the map

$$
\mathrm{e}^{B}: \mathbb{T} M \rightarrow \mathbb{T} M, \quad \mathrm{e}^{B}:=\left(\begin{array}{cc}
1 & 0 \\
B^{b} & 1
\end{array}\right)
$$

The $B$-transform $\mathrm{e}^{B}$ is called closed or exact if the two-form $B$ is closed or exact, respectively.

Proposition 2.5. Let $M$ be a manifold and let $B \in \Omega^{2}(M)$. The B-field transform $\mathrm{e}^{B}$ is orthogonal with respect to the standard metrics on $\mathbb{T} M$ and $\mathbb{T}_{\mathbb{C}} M$. It transforms almost $G C$ structures on $V$ by

$$
\mathcal{J} \mapsto \mathrm{e}^{B} \circ \mathcal{J} \circ \mathrm{e}^{-B} \quad \text { and } \quad E \mapsto \mathrm{e}^{B}(E)
$$

for an almost $G C$ structure given equivalently by a map $\mathcal{J}$ or a Dirac structure $E$, and it preserves types. It transforms almost $G K$ structures $\left(\mathcal{J}_{1}, \mathcal{J}_{2}\right)$ by transforming $\mathcal{J}_{1}$ and $\mathcal{J}_{2}$ individually.

The Lie bracket defines a skew-symmetric bilinear bracket on sections of the tangent bundle $T M$. This can be extended to a skew-symmetric bilinear bracket on sections of the Pontryagin bundle $\mathbb{T} M$, called the Courant bracket, defined by

$$
[X+\alpha, Y+\beta]:=[X, Y]+\mathcal{L}_{X} \beta-\mathcal{L}_{Y} \alpha-\frac{1}{2} \mathrm{~d}(\beta(X)-\alpha(Y))
$$

for all $X+\alpha, Y+\beta \in \Gamma(\mathbb{T} M)$, where $\Gamma(\mathbb{T} M)$ denotes the space of smooth sections of $\mathbb{T} M \rightarrow M$. Here the bracket on the right-hand side is the usual Lie bracket of vector fields, and $\mathcal{L}$ denotes Lie differentiation. For each closed differential three-form $H \in \Omega_{\mathrm{cl}}^{3}(M)$, there is also the $H$-twisted Courant bracket, defined by

$$
[X+\alpha, Y+\beta]_{H}:=[X+\alpha, Y+\beta]+\iota_{Y} \iota_{X} H
$$

$X+\alpha, Y+\beta \in \Gamma(\mathbb{T} M)$. Both the Courant and the $H$-twisted Courant brackets extend complex linearly to brackets on smooth sections of the complexified Pontryagin bundle $\mathbb{T}_{\mathbb{C}} M$, which will be denoted the same way.

Definition 2.6. Let $M$ be a manifold, and let $L$ be a real (respectively complex) linear subbundle of $\mathbb{T} M$ (respectively $\mathbb{T}_{\mathbb{C}} M$ ). Then $L$ is Courant involutive if the space $\Gamma(L)$ of smooth sections of $L$ is closed under the Courant bracket, i.e. $[\Gamma(L), \Gamma(L)] \subset \Gamma(L)$. If $H \in \Omega_{\mathrm{cl}}^{3}(M)$, we similarly define $H$-twisted Courant involutive. 
Let $E \subset \mathbb{T}_{\mathbb{C}} M$ be an almost $\mathrm{GC}$ structure on $M$. This is a $\boldsymbol{G C}$ structure if $E$ is Courant involutive, in which case $(M, E)$ is a $\boldsymbol{G C}$ manifold. If $H \in \Omega_{\mathrm{cl}}^{3}(M)$ and $E$ is $H$-twisted Courant involutive, then $E$ is an $H$-twisted $\boldsymbol{G C}$ structure, and $(M, E, H)$ is a twisted $G C$ manifold.

Let $\left(\mathcal{J}_{1}, \mathcal{J}_{2}\right)$ be an almost GK structure on $M$. This is a $\boldsymbol{G K}$ structure if both $\mathcal{J}_{1}$ and $\mathcal{J}_{2}$ are Courant involutive, in which case $\left(M, \mathcal{J}_{1}, \mathcal{J}_{2}\right)$ is a $\boldsymbol{G K}$ manifold. If $H \in \Omega_{\mathrm{cl}}^{3}(M)$ and $\mathcal{J}_{1}$ and $\mathcal{J}_{2}$ are $H$-twisted Courant involutive, then this is an $H$-twisted $\boldsymbol{G K}$ structure, and $\left(M, \mathcal{J}_{1}, \mathcal{J}_{2}, H\right)$ is a twisted GK manifold.

Remark 2.7. Let $M$ be a manifold and $D$ be a real or complex Dirac structure on $M$. Then $D$ is called closed, or integrable, if the space $\Gamma(D)$ of smooth sections of $D$ is Courant involutive. Thus a GC structure on $M$ is a closed complex Dirac structure $E \subset \mathbb{T}_{\mathbb{C}} M$ such that $E \cap \bar{E}=0$.

Proposition 2.8 (Proposition 3.23 of [9]). Let $M$ be a manifold, let $H \in \Omega_{c l}^{3}(M)$, and let $B \in \Omega^{2}(M)$. The $B$-transform of an $H$-twisted $G C$ structure is an $(H+\mathrm{d} B)$-twisted $G C$ structure. Thus, a closed B-transform of an untwisted GC structure is untwisted.

\section{Example 2.9.}

1. Let $(M, \omega)$ be an almost symplectic manifold, meaning that $\omega \in \Omega^{2}(M)$ is a nondegenerate form on $M$, but not necessarily closed. This defines an almost GC structure $\mathcal{J}_{\omega}$ on $M$ by

$$
\mathcal{J}_{\omega}:=\left(\begin{array}{cc}
0 & -\omega^{\sharp} \\
\omega^{b} & 0
\end{array}\right)
$$

of type 0 at every point. It has associated Dirac structure defined by

$$
E_{\omega, x}=\left\{X-i \omega_{x}^{b}(X) \mid X \in T_{\mathbb{C}, x} M\right\}
$$

for each $x \in M$. As discussed in Section 3 of [9], it is a GC structure if and only if $\mathrm{d} \omega=0$, i.e. if and only if $\omega$ is a symplectic structure on $M$.

2. Let $(M, I)$ be an almost complex manifold, meaning that $I^{2}=-\mathrm{id}_{T M}$ but $I$ is not necessarily integrable. This defines an almost GC structure $\mathcal{J}_{I}$ on $M$ by

$$
\mathcal{J}_{I}:=\left(\begin{array}{cc}
-I & 0 \\
0 & I^{*}
\end{array}\right)
$$

of type $n$ at every point. It has associated Dirac structure defined by

$$
E_{I}=T_{0,1} M \oplus T_{1,0}^{*} M
$$

where $T_{1,0} M, T_{0,1} M \subset T_{\mathbb{C}} M$ denote the $\pm i$-eigenbundles of $I$. As discussed in Section 3 of [9], it is a GC structure if and only if $I$ is integrable, i.e. if and only if $I$ is a complex structure on $M$.

3. Let $M$ be a Kähler manifold with Kähler form $\omega \in \Omega^{2}(M)$, complex structure $I: T M \rightarrow$ $T M$, and associated Riemannian metric $g$. Then $\mathcal{J}_{\omega}$ and $\mathcal{J}_{I}$ commute and

$$
G:=-\mathcal{J}_{\omega} \circ \mathcal{J}_{I}=\left(\begin{array}{cc}
0 & g^{\sharp} \\
g^{b} & 0
\end{array}\right)
$$

is positive definite, so $\left(M, \mathcal{J}_{\omega}, \mathcal{J}_{I}\right)$ is a GK manifold. 
Example 2.10. Let $\left(M_{1}, \mathcal{J}_{1}\right)$ and $\left(M_{2}, \mathcal{J}_{2}\right)$ be almost GC manifolds. Then the direct sum $\mathcal{J}$ of $\mathcal{J}_{1}$ and $\mathcal{J}_{2}$ is a map $\mathcal{J}:=\left(\mathcal{J}_{1}, \mathcal{J}_{2}\right): \mathbb{T} M_{1} \oplus \mathbb{T} M_{2} \rightarrow \mathbb{T} M_{1} \oplus \mathbb{T} M_{2}$, which under the identification $\mathbb{T} M_{1} \oplus \mathbb{T} M_{2} \cong \mathbb{T}\left(M_{1} \times M_{2}\right)$ yields an almost GC structure on $M_{1} \times M_{2}$. We will call this the direct sum of the almost GC structures on $M_{1}$ and $M_{2}$. It is not hard to see that $\left(\mathcal{J}_{1}, \mathcal{J}_{2}\right)$ is a GC structure on $M_{1} \times M_{2}$ if and only if $\mathcal{J}_{i}$ is a GC structure on $M_{i}$ for $i=1,2$.

Let $H_{1} \in \Omega_{\mathrm{cl}}^{3}\left(M_{1}\right)$ and $H_{2} \in \Omega_{\mathrm{cl}}^{3}\left(M_{2}\right)$, let $\pi_{i}: M_{1} \times M_{2} \rightarrow M_{i}$ be the natural projection for $i=1,2$, and set $H:=\pi_{1}^{*} H_{1}+\pi_{2}^{*} H_{2}$. By the naturality of the exterior derivative, we know $H$ is a closed three-form on $M_{1} \times M_{2}$. Furthermore, it is not hard to see that $\left(\mathcal{J}_{1}, \mathcal{J}_{2}\right)$ is an $H$-twisted GC structure on $M_{1} \times M_{2}$ if and only if $\mathcal{J}_{i}$ is an $H_{i}$-twisted GC structure on $M_{i}$ for $i=1,2$.

There is a completely analogous product construction for almost GK and GK manifolds as well.

Let $(M, E, H)$ be a twisted GC manifold. Suppose $S$ is a submanifold of $M$ given by the embedding $j: S \hookrightarrow M$. Although $j$ induces a natural embedding $j_{*}: T S \hookrightarrow T M$ of tangent bundles, because of the contravariance of cotangent bundles there is in general no obvious embedding $\mathbb{T} S \hookrightarrow \mathbb{T} M$ of the Pontryagin bundles. The following definition comes from [3].

For each $x \in S$, define

$$
E_{S, x}:=\left\{\left(X,\left.\lambda\right|_{S}\right) \in \mathbb{T}_{\mathbb{C}, x} S \mid(X, \lambda) \in\left(T_{\mathbb{C}, x} S \oplus T_{\mathbb{C}, x}^{*} M\right) \cap E_{x}\right\}
$$

By [3, Lemma 8.2], this $E_{S, x}$ is a maximally isotropic complex subspace of $\mathbb{T}_{\mathbb{C}, x} S$. Let $E_{S}:=$ $\bigsqcup_{x \in S} E_{S, x}$. Then $E_{S}$ is a constant-rank complex linear distribution of $\mathbb{T}_{\mathbb{C}} S$, but is not in general a smooth subbundle, nor will it generally satisfy $E_{S} \cap \overline{E_{S}}=0$.

Proposition 2.11. Let $(M, E, H)$ be a twisted $G C$ manifold, let $j: S \hookrightarrow M$ be a submanifold, and let $E_{S} \subset \mathbb{T}_{\mathbb{C}} S$ be as defined above. If $E_{S}$ is a subbundle of $\mathbb{T}_{\mathbb{C}} S$, then $E_{S}$ is $\left(j^{*} H\right)$-twisted Courant involutive.

In the untwisted case, where $H=0$, Proposition 2.11 was proved in [5, Corollary 3.1.4]. The proof in the twisted case is nearly identical, with only minor changes to this proof and the relevant definitions and precursory results, (i.e. Definition 2.3.2, Propositions 2.3.3 and 3.1.3, and Corollary 3.1.4 in [5]).

Definition 2.12. Let $(M, E, H)$ be a twisted GC manifold, and let $j: S \hookrightarrow M$ be a submanifold. If $E_{S} \subset \mathbb{T}_{\mathbb{C}} S$ is a subbundle and satisfies $E_{S} \cap \overline{E_{S}}=0$, then $\left(S, E_{S}, j^{*} H\right)$ is a (twisted) $\boldsymbol{G C}$ submanifold of $(M, E, H)$, and we denote by $\mathcal{J}_{S}$ the GC structure on $S$ induced by $E_{S}$.

Remark 2.13. Suppose $(M, E, H)$ is a twisted GC manifold, and $j: S \hookrightarrow M$ is an open submanifold. Then since $T S$ and $T^{*} S$ can be identified with $\left.(T M)\right|_{S}$ and $\left.\left(T^{*} M\right)\right|_{S}$, respectively, we see that we can identify $E_{S}$ with $\left.E\right|_{S}$, that $\mathcal{J}_{S}=\left.\mathcal{J}\right|_{\mathbb{T} S}$, and that $j^{*} H$ can be identified with $\left.H\right|_{S}$. Therefore an open submanifold of an $H$-twisted GC manifold is automatically an $H$-twisted GC manifold. Similarly, an open submanifold of an $H$-twisted GK manifold is automatically an $H$-twisted GK manifold.

Definition 2.14. Let $(M, \mathcal{J})$ be an almost GC manifold, and let $S \subset M$ be a submanifold. A splitting bundle for $S$ with respect to $(M, \mathcal{J})$ is a subbundle $N$ of $\left.T M\right|_{S} \rightarrow S$ such that $\left.T M\right|_{S}=T S \oplus N$ and $T S \oplus \operatorname{Ann}(N) \subset \mathbb{T} M$ is invariant under $\mathcal{J}$. If a splitting bundle exists for $S$, then $S$ is called a split submanifold of $(M, \mathcal{J})$.

The following is an extension of Proposition 5.12 of [3] to the twisted case. As with Proposition 2.11, the original proof still holds with only minor alterations. 
Proposition 2.15. Let $(M, \mathcal{J}, H)$ be a twisted $G C$ manifold, and let $i: S \hookrightarrow M$ be a split submanifold of $M$ with splitting bundle $N \rightarrow S$. Then $S$ is an $\left(i^{*} H\right)$-twisted $G C$ submanifold of $(M, \mathcal{J}, H)$, and the $G C$ structure corresponding to the bundle $E_{S}$ is the same as the one induced by the restriction of $\mathcal{J}$ via the natural isomorphism

$\mathbb{T} S \cong T S \oplus \operatorname{Ann} N \subset \mathbb{T} M$

It is straightforward to show that this implies the following.

Corollary 2.16. Let $\left(M, \mathcal{J}_{1}, \mathcal{J}_{2}, H\right)$ be a twisted $G K$ manifold, and and let $i: S \hookrightarrow M$ be a split submanifold of $M$ with respect to both $\mathcal{J}_{1}$ and $\mathcal{J}_{2}$, with common splitting bundle $N \rightarrow S$. Then $\left(S,\left(\mathcal{J}_{1}\right)_{W},\left(\mathcal{J}_{2}\right)_{W}\right)$ is an $\left(i^{*} H\right)$-twisted $G K$ manifold.

Definition 2.17. Let $M$ be a manifold, and let $G$ be a Lie group acting smoothly on $M$. This lifts to an action of $G$ on $\mathbb{T} M$ by bundle automorphisms, given by

$$
\left(g_{*},\left(g^{-1}\right)^{*}\right): \mathbb{T} M \rightarrow \mathbb{T} M
$$

for each $g \in G$, where $g_{*}$ is the pushforward of tangent vectors by the map $g: M \rightarrow M$ and $\left(g^{-1}\right)^{*}$ is the pullback of tangent covectors by the map $g^{-1}: M \rightarrow M$.

Let $\mathcal{J}$ be an $H$-twisted GC structure on $M$. We say that the $G$-action on $(M, \mathcal{J}, H)$ is canonical if the following hold.

1. The differential form $H$ is $G$-invariant, i.e. $g^{*} H=H$ for all $g \in G$.

2. The action of $G$ on $\mathbb{T} M$ commutes with $\mathcal{J}$, i.e. the diagram

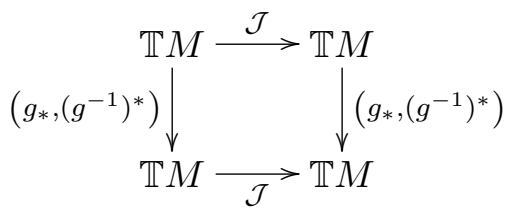

commutes for all $g \in G$.

It is easy to check that a smooth group action on a manifold commutes with an almost GC structure $\mathcal{J}: \mathbb{T} M \rightarrow \mathbb{T} M$ if and only if the complex linear extension of the action preserves the corresponding complex Dirac structure.

Example 2.18. Let $(M, \omega)$ be an almost symplectic manifold, let $\omega^{b}: T M \rightarrow T^{*} M$ be the associated bundle isomorphism, and let

$$
\mathcal{J}_{\omega}:=\left(\begin{array}{cc}
0 & -\omega^{\sharp} \\
\omega^{b} & 0
\end{array}\right)
$$

be the associated almost GC structure on $M$. Let $G$ be a Lie group acting smoothly on $M$. It is easy to check that the $G$-action is symplectic if and only if the map $\omega^{b}: T M \rightarrow T^{*} M$ is $G$-equivariant with respect to the pushforward action on $T M$ and the inverse pullback action on $T^{*} M$, if and only if the $G$-action commutes with $\mathcal{J}_{\omega}$.

Recall that for a smooth action of a compact Lie group $G$ on a manifold $M$, each connected component of the fixed point set $M^{G}$ is a closed submanifold of $M$. (Different components of $M^{G}$ may have different dimensions.)

Proposition 2.19. Let $M$ be a manifold, and let $\mathcal{J}$ be an almost $G C$ structure on $M$. Suppose the compact Lie group $G$ acts canonically on $(M, \mathcal{J})$. Then each component of $M^{G}$ is a split submanifold of $(M, \mathcal{J})$. 
Proof. Let $\left(M^{G}\right)^{\prime}$ be a component of $M^{G}$. First, recall that for each $x \in\left(M^{G}\right)^{\prime}$ the derivative of the action of $G$ at $x$ defines a linear action of $G$ on $T_{x} M$, and that $T_{x}\left(M^{G}\right)^{\prime}=\left(T_{x} M\right)^{G}$. Let $d g$ be a bi-invariant Haar measure on $G$, adjusted so that $d g(G)=1$. Define a bundle map $\pi:\left.(T M)\right|_{\left(M^{G}\right)^{\prime}} \rightarrow T\left(M^{G}\right)^{\prime}$ by setting

$$
\pi_{x}(v):=\int_{G}(g \cdot v) d g \quad \text { for all } \quad v \in V,
$$

for each $x \in\left(M^{G}\right)^{\prime}$. Define the subbundle $\left.N \subset(T M)\right|_{\left(M^{G}\right)^{\prime}}$ by setting $N_{x}:=\operatorname{ker} \pi_{x}$ for each $x \in\left(M^{G}\right)^{\prime}$. That $\pi$ is a bundle map and $N$ is a vector bundle follow from the naturality of the technique of averaging by integration. Note also that $T_{x} M=\left(T_{x} M\right)^{G} \oplus N_{x}=T_{x}\left(M^{G}\right)^{\prime} \oplus N_{x}$ for each $x \in\left(M^{G}\right)^{\prime}$. To conclude that $N$ is a splitting for $\left(M^{G}\right)^{\prime} \subset(M, \mathcal{J})$, it remains only to show that $T\left(M^{G}\right)^{\prime} \oplus \operatorname{Ann}(N)=\left.(T M)^{G}\right|_{\left(M^{G}\right)^{\prime}} \oplus \operatorname{Ann}(N)$ is preserved by $\mathcal{J}$.

Fix $x \in\left(M^{G}\right)^{\prime}$, let $V=T_{x} M$, and let $W=N_{x}$. We claim that $\operatorname{Ann}(W)=\left(V^{*}\right)^{G}$, which would imply that $\left(V \oplus V^{*}\right)^{G}=V^{G} \oplus\left(V^{*}\right)^{G}=V^{G} \oplus \operatorname{Ann}(W)$. Since $\mathcal{J}_{x}$ commutes with the action of $G$ on $V \oplus V^{*}$, we know that $\mathcal{J}\left(\left(V \oplus V^{*}\right)^{G}\right)=\left(V \oplus V^{*}\right)^{G}$, and hence a proof of this claim completes the proof of this proposition.

Let $\lambda \in \operatorname{Ann}(W)$, let $g \in G$, and let $u \in V$. Decompose $u$ as $u=v+w$ for $v \in V^{G}$ and $w \in W$. Then

$$
\begin{array}{rlrl}
(g \cdot \lambda)(u) & =\lambda\left(g^{-1} \cdot u\right) & & \\
& =\lambda\left(g^{-1} \cdot v\right)+\lambda\left(g^{-1} \cdot w\right) & \\
& =\lambda(v)+\lambda\left(g^{-1} \cdot w\right) & & \text { since } v \in V^{G} \\
& =\lambda(v)+0 & & \text { since } w \in W, W \text { is } G \text {-stable, and } \lambda \in \operatorname{Ann}(W) \\
& =\lambda(v)+\lambda(w) & & \text { since } w \in W \text { and } \lambda \in \operatorname{Ann}(W) \\
& =\lambda(u) . & &
\end{array}
$$

Therefore $\lambda \in\left(V^{*}\right)^{G}$, so $\operatorname{Ann}(W) \subset\left(V^{*}\right)^{G}$. Note that

$$
\operatorname{Ann}(W) \cong(V / W)^{*}=\left(\left(V^{G} \oplus W\right) / W\right)^{*} \cong\left(V^{G}\right)^{*} .
$$

Hence $\operatorname{dim} \operatorname{Ann}(W)=\operatorname{dim}\left(V^{G}\right)$, so $\operatorname{Ann}(W)=\left(V^{*}\right)^{G}$.

\section{Background information on $G$-spaces}

In this section we give some brief definitions and results about compact group actions on manifolds which will be required in later sections. The standard reference for the material on equivariant cohomology is [10]. The material on orbit spaces and their stratification by orbit types can be found in [7, Chapter 2] and [18, Chapter 2].

\subsection{Equivariant cohomology}

Let $M$ be a manifold and $G$ be a compact Lie group acting smoothly on $M$. Consider the space $\Omega^{k}(M) \otimes S^{i}\left(\mathfrak{g}^{*}\right)$, where $S^{i}$ denotes the degree $i$ elements of the symmetric algebra. This is a $G$ space with action defined by linear extension of the rule $g \cdot(\alpha \otimes p):=\left(\left(g^{-1}\right)^{*} \alpha\right) \otimes\left(p \circ \operatorname{Ad}_{g^{-1}}\right)$ for $g \in G, \alpha \in \Omega^{\star}(M), p \in S\left(\mathfrak{g}^{*}\right)$. We can identify $\Omega^{k}(M) \otimes S^{i}\left(\mathfrak{g}^{*}\right)$ with the space of degree $i$ polynomial maps $\mathfrak{g} \rightarrow \Omega^{k}(M)$ via

$$
\alpha \otimes p: \xi \mapsto p(\xi) \cdot \alpha
$$

for $\xi \in \mathfrak{g}$. An element of $\Omega^{k}(M) \otimes S^{i}\left(\mathfrak{g}^{*}\right)$ is $G$-invariant if and only if its corresponding polynomial map is $G$-equivariant with respect to the adjoint action of $G$ on $\mathfrak{g}$ and the action of $G$ on $\Omega^{k}(M)$ given by $g \cdot \alpha:=\left(g^{-1}\right)^{*} \alpha$ for $g \in G, \alpha \in \Omega^{k}(M)$. 
Definition 3.1. Let $M$ be a manifold and $G$ be a compact Lie group acting smoothly on $M$. The space of equivariant differential forms of degree $n$ on $M$ is

$$
\Omega_{G}^{n}(M):=\bigoplus_{i=0}^{\lfloor n / 2\rfloor}\left(\Omega^{n-2 i}(M) \otimes S^{i}\left(\mathfrak{g}^{*}\right)\right)^{G} .
$$

The differential $\mathrm{d}_{G}: \Omega_{G}^{n} \rightarrow \Omega_{G}^{n+1}$ is defined, viewing equivariant forms as maps $\mathfrak{g} \rightarrow \Omega^{\star}(M)$, by

$$
\mathrm{d}_{G}(\alpha \otimes p)(\xi):=\left(\mathrm{d} \alpha-\iota_{\xi_{M}} \alpha\right) p(\xi) \text { for all } \xi \in \mathfrak{g} .
$$

The Cartan model for the G-equivariant cohomology of $M$ is $H_{G}^{\star}(M):=H^{\star}\left(\Omega_{G}^{\star}, \mathrm{d}_{G}\right)$.

Suppose now that $G$ acts freely on $M$. Then the $G$-equivariant cohomology of $M$ is naturally isomorphic as a graded algebra to the de Rham cohomology of the quotient $M / G$,

$$
H_{G}^{\star}(M) \cong H^{\star}(M / G) .
$$

We denote this isomorphism by $\kappa: H_{G}^{\star}(M) \rightarrow H^{\star}(M / G)$.

Let $B \in \Omega^{n}(M)$. The form $B$ is called basic if it is $G$-equivariant and if $\iota_{\xi_{M}} B=0$ for all $\xi \in \mathfrak{g}$. If there is a differential form $\widetilde{B} \in \Omega^{n}(M / G)$ such that the pullback of $\widetilde{B}$ by the quotient map $M \rightarrow M / G$ equals $B$, then we say $B$ descends to $\widetilde{B}$.

Proposition 3.2. Let $M$ be a manifold and $G$ be a compact Lie group acting smoothly and freely on $M$.

(a) If $B \in \Omega^{n}(M)$ is basic, then $B$ descends to some $\widetilde{B} \in \Omega^{n}(M / G)$.

(b) If $B \in \Omega^{n}(M)^{G} \subset \Omega_{G}^{n}(M)$ is equivariantly closed, i.e. $\mathrm{d}_{G} B=0$, then $B$ is closed and basic and descends to some closed $\widetilde{B} \in \Omega^{n}(M / G)$ such that

$$
\kappa[B]=[\widetilde{B}]
$$

where $[B]$ and $[\widetilde{B}]$ are the cohomology classes of $B$ and $\widetilde{B}$, respectively.

(c) If $\eta \in \Omega_{G}^{n}(M)$ is equivariantly closed, then there exists $\Gamma \in \Omega_{G}^{n-1}(M)$ so that $\eta+\mathrm{d}_{G} \Gamma \in$ $\Omega^{n}(M)^{G} \subset \Omega_{G}^{n}(M)$. In this case, since $\eta+\mathrm{d}_{G} \Gamma$ is equivariantly closed, it descends to some $\widetilde{\eta} \in \Omega^{n}(M / G)$ such that $\kappa[\eta]=[\widetilde{\eta}]$.

Definition 3.3. Let $M$ be a manifold and $G$ be a compact Lie group acting on $M$ smoothly and freely. Then $M \rightarrow M / G$ is a (left) principal $G$-bundle. A connection on this bundle is a $\mathfrak{g}$-valued one-form $\theta \in \Omega^{1}(M, \mathfrak{g})$ such that

1. $\theta$ is $G$-equivariant, i.e. $g^{*} \theta=\operatorname{Ad}_{g} \circ \theta$;

2. $\theta\left(\xi_{M}\right) \equiv \xi$ for all $\xi \in \mathfrak{g}$.

\subsection{Orbit type stratification}

Let $G$ be a group. For each subgroup $H$ of $G$, we will denote by $(H)$ the set of subgroups of $G$ that are conjugate to $H$. Suppose $G$ is a compact Lie group and $M$ is a manifold on which $G$ acts smoothly. Note that the conjugacy relation among subgroups of $G$ preserves closedness, and hence also preserves the property of being a Lie subgroup.

Definition 3.4. Let $x \in M$, and let $G_{x}:=\{g \in G \mid g \cdot x=x\}$ be the isotropy subgroup of $x$ in $G$. The orbit type of the point $x$, or of $G \cdot x$, is the set $\left(G_{x}\right)$ of subgroups of $G$ that are conjugate to $G_{x}$.

Let $H$ be a closed subgroup $H$ of $G$. The $(H)$-orbit type submanifold of $M$ is the set $M_{(H)}:=\left\{x \in M \mid G_{x} \in(H)\right\}$. The $H$-isotropy type submanifold of $M$ is the set $M_{H}:=\{x \in$ $\left.M \mid G_{x}=H\right\}$. The $H$-fixed point submanifold of $M$ is the set $M^{H}:=\left\{x \in M \mid G_{x} \subset H\right\}$. 
Note that the sets defined above are related by the equation $M_{H}=M_{(H)} \cap M^{H}$. Also, two $G$-orbits in $M$ have the same orbit type if and only if they are $G$-equivariantly diffeomorphic. This leads one to the following definitions.

Definition 3.5. Let $x \in M$, and let $H=G_{x}$. The local action type submanifold through $x$ is the subset $M_{(H)}^{l_{x}} \subset M$ of points $y \in M$ such that there is a $G$-equivariant diffeomorphism between $G$-invariant open neighborhoods of $x$ and $y$. Define $M_{H}^{l_{x}}:=M_{(H)}^{l_{x}} \cap M^{H}$.

Some important properties of the sets we have defined above are collected in the following proposition. Their proofs can be found in the references cited at the beginning of this section.

Proposition 3.6. Let $G$ be a compact Lie group, and $M$ be a manifold on which $G$ acts smoothly. Let $x \in M$ and put $H=G_{x}$. Then the following hold.

(a) $M_{(H)}^{l_{x}}$, respectively $M_{H}^{l_{x}}$, is an open and closed subset of $M_{(H)}$, respectively $M_{H}$.

(b) The sets $M_{(H)}^{l_{x}}$ and $M_{H}^{l_{x}}$ are locally closed embedded submanifolds of $M$, as is each connected component of $M_{H}$, of $M_{(H)}$, and of $M^{H}$.

(c) $M_{H}^{l_{x}}$ and $M_{(H)}^{l_{x}}$ consists of the union of certain components of $M_{H}$ and $M_{(H)}$, respectively.

(d) $M_{H}$ and $M_{H}^{l_{x}}$ are open in $M^{H}$.

(e) $M_{(H)}$ and $M_{(H)}^{l_{x}}$ are $G$-stable, and $G \cdot M_{H}=M_{(H)}$ and $G \cdot M_{H}^{l_{x}}=M_{(H)}^{l_{x}}$.

(f) Let $N=N_{G}(H)$ be the normalizer of $H$ in $G$. Both $M_{H}$ and $M_{H}^{l_{x}}$ are $N$-stable, and $N / H$ acts freely on both. Hence $M_{H}^{l_{x}} / N \cong M_{H}^{l_{x}} /(N / H)$ is a manifold.

(g) The inclusions $M_{H} \hookrightarrow M_{(H)}$ and $M_{H}^{l_{x}} \hookrightarrow M_{(H)}^{l_{x}}$ induce homeomorphisms $M_{H} \rightarrow M_{(H)}$ and $M_{H}^{l_{x}} / N \rightarrow M_{(H)}^{l_{x}} / G$. Thus the quotient $M_{(H)}^{l_{x}} / G$ inherits a natural manifold structure.

(h) Each component of the quotient $M_{(H)} / G$ inherits a natural manifold structure.

In general, the orbit space $M / G$ can be a very singular space. It will be a Hausdorff and second-countable topological space, but will rarely inherit a manifold, or even an orbifold, structure from $M$. However, because $M$ is the disjoint union of its orbit type submanifolds, we can also partition the orbit space:

$$
M / G=\bigsqcup_{(H)} M_{(H)} / G,
$$

where the disjoint union is taken over all the distinct orbit type submanifolds of $M$. Since each component of $M_{(H)} / G$ is a manifold, we know that, after refining the partition to components, (3.1) is a partition of $M / G$ into manifolds. It is called the orbit type partition of $M / G$.

Remark 3.7. All of the above results hold true even if $G$ is an arbitrary Lie group, so long as it acts on $M$ both smoothly and properly.

\section{Hamiltonian actions on generalized complex manifolds}

In [14], the authors proposed the following definition of Hamiltonian actions on GC manifolds.

Definition 4.1. Let $(M, \mathcal{J})$ be an untwisted GC manifold, let $E$ be the associated complex Dirac structure on $M$, and let $G$ be a Lie group acting canonically on $(M, \mathcal{J})$. This action is 
generalized Hamiltonian if there exists a $G$-equivariant map $\mu: M \rightarrow \mathfrak{g}^{*}$ such that, for all $\xi \in \mathfrak{g}$,

$$
\xi_{M}=-\mathcal{J}\left(\mathrm{d} \mu^{\xi}\right)
$$

or equivalently $\xi_{M}-i \mathrm{~d} \mu^{\xi} \in \Gamma(E)$. Here $\mu^{\xi}: M \rightarrow \mathbb{R}$ is the smooth function defined by $\mu^{\xi}(x):=$ $\langle\mu(x), \xi\rangle$ for all $x \in M$. The map $\mu$ is called a generalized moment map for the $G$-action on $(M, \mathcal{J})$.

Let $(M, \mathcal{J}, H)$ be a twisted GC manifold, and let $G$ be a Lie group acting canonically on $(M, \mathcal{J}, H)$. This action is twisted generalized Hamiltonian if there exists a $G$-equivariant map $\mu: M \rightarrow \mathfrak{g}^{*}$ and a $G$-equivariant $\mathfrak{g}^{*}$-valued one-form $\alpha \in \Omega^{1}\left(M, \mathfrak{g}^{*}\right)$ on $M$ such that, for all $\xi \in M$,

1. $\xi_{M}=-\mathcal{J}\left(\mathrm{d} \mu^{\xi}\right)-\alpha^{\xi}$ (or equivalently $\xi_{M}+\alpha^{\xi}-i \mathrm{~d} \mu^{\xi} \in \Gamma(E)$ ), and

2. $\iota_{\xi_{M}} H=\mathrm{d} \alpha^{\xi}$.

Here $\mu^{\xi}$ is as defined above, and $\alpha^{\xi} \in \Omega^{1}(M)$ is the differential one-form on $M$ defined by $\left(\alpha^{\xi}\right)_{x}(v):=\left\langle\alpha_{x}(v), \xi\right\rangle$ for all $x \in M, v \in T_{x} M$. The map $\mu$ and the one-form $\alpha$ are called a generalized moment map and a moment one-form, respectively, for the action $G$-action on $(M, \mathcal{J}, H)$.

Definition 4.2. Let $\left(M, \mathcal{J}_{1}, \mathcal{J}_{2}\right)$ be a GK manifold, and let $G$ be a Lie group acting on $M$ and preserving both $\mathcal{J}_{1}$ and $\mathcal{J}_{2}$. This action is called generalized Hamiltonian if the action of $G$ on $\left(M, \mathcal{J}_{1}\right)$ is generalized Hamiltonian.

Similarly, if $\left(M, \mathcal{J}_{1}, \mathcal{J}_{2}, H\right)$ be a twisted GK manifold, and the $G$-action preserves $\mathcal{J}_{1}, \mathcal{J}_{2}$, and $H$, then the action is twisted generalized Hamiltonian if the action of $G$ on $\left(M, \mathcal{J}_{1}, H\right)$ is twisted generalized Hamiltonian.

\section{Remark 4.3.}

(a) Note that a moment one-form $\alpha \in \Omega^{1}\left(M, \mathfrak{g}^{*}\right)$ is an equivariant differential form of degree 3 .

(b) Because $E$ is an isotropic subbundle, the condition that $\xi_{M}+\alpha^{\xi}-i \mathrm{~d} \mu^{\xi} \in E$ implies that $\left\langle\left\langle\xi_{M}+\alpha^{\xi}-i \mathrm{~d} \mu^{\xi}, \xi_{M}+\alpha^{\xi}-i \mathrm{~d} \mu^{\xi}\right\rangle\right\rangle=0$, and hence that $\iota_{\xi_{M}} \alpha^{\xi}=\iota_{\xi_{M}} \mathrm{~d} \mu^{\xi}=0$.

(c) Given a GC manifold $(M, \mathcal{J})$, one can consider this as a twisted GC manifold $(M, \mathcal{J}, H)$ by setting $H=0$. Therefore, if a Lie group $G$ acts on $(M, \mathcal{J})$ canonically, we have two notions of whether the action is Hamiltonian. It may be Hamiltonian as an action on $(M, \mathcal{J})$, in which case there is just a moment map, or it may be Hamiltonian as an action on $(M, \mathcal{J}, H)$, in which case there is both a moment map and a moment one-form. It is potentially interesting to explore both possibilities.

Example 4.4. Let $(M, \omega)$ be a symplectic manifold, and let $G$ be a Lie group acting on $(M, \omega)$ in a Hamiltonian fashion with moment map $\Phi: M \rightarrow \mathfrak{g}^{*}$. Recall that this means the $G$-action is symplectic, the map $\Phi$ is $G$-equivariant, and for all $\xi \in \mathfrak{g}$ we have $\mathrm{d} \Phi^{\xi}=\iota_{\xi_{M}}$. Let $\mathcal{J}_{\omega}$ be the GC structure on $M$ induced by $\omega$. As discussed in Example 3.8 of [14], the action of $G$ on $\left(M, \mathcal{J}_{\omega}\right)$ is generalized Hamiltonian, and $\Phi$ is a generalized moment map.

Theorem 4.5. Let $(M, E, H)$ be a twisted $G C$ manifold, where $E$ is the associated complex Dirac structure, and let $G$ be a Lie group acting on $(M, E, H)$ in a Hamiltonian fashion with moment map $\mu: M \rightarrow \mathfrak{g}^{*}$ and moment one-form $\alpha \in \Omega^{1}\left(M, \mathfrak{g}^{*}\right)$. If $j: S \hookrightarrow M$ is a $G$-stable twisted $G C$ submanifold of $(M, E, H)$, then the restriction of the action of $G$ to $\left(S, E_{S}, j^{*} H\right)$ is Hamiltonian with moment map $\left.\mu\right|_{S}: S \rightarrow \mathfrak{g}^{*}$ and moment one-form $j^{*} \alpha \in \Omega^{1}\left(S, \mathfrak{g}^{*}\right)$. 
Proof. First we will prove that the action of $G$ on $S$ preserves $E_{S}$. Let $x \in S$ and $(X, \lambda) \in$ $\left(T_{\mathbb{C}, x} S \oplus T_{\mathbb{C}, x}^{*} M\right) \cap E_{x}$, which means that $\left(X,\left.\lambda\right|_{S}\right) \in E_{S, x}$. Then for any $g \in G$ we have

$$
g \cdot\left(X+j^{*} \lambda\right)=g_{*}(X)+\left.\left(g^{-1}\right)^{*} \lambda\right|_{S} .
$$

Because $S$ is $G$-stable, the inclusion $j: S \hookrightarrow M$ is $G$-equivariant, i.e. the $G$-action commutes with $j$. Hence $j_{*}: T S \hookrightarrow T M$ is $G$-equivariant, so $g_{*}(X) \in T_{g \cdot x} S$. Also

$$
\left(g^{-1}\right)^{*}\left(\left.\lambda\right|_{S}\right)=\left(g^{-1}\right)^{*} j^{*} \lambda=j^{*}\left(g^{-1}\right)^{*} \lambda=\left.\left(\left(g^{-1}\right)^{*} \lambda\right)\right|_{S} .
$$

Since $E$ is $G$-stable, we have $g \cdot(X+\lambda)=g_{*}(X)+\left(g^{-1}\right)^{*} \lambda \in E_{g \cdot x}$. Therefore $g \cdot\left(X, j^{*} \lambda\right)=$ $\left(g_{*}(X), j^{*}\left(g^{-1}\right)^{*} \lambda\right) \in E_{S, g \cdot x}$. Thus $E_{S}$ is $G$-stable.

Now suppose that $\left(S, E_{S}, j^{*} H\right)$ is a GC submanifold of $(M, E, H)$, meaning that $E_{S}$ is a vector bundle, that $E_{S} \cap \overline{E_{S}}=0$, and that $E_{S}$ is $j^{*} H$-twisted Courant involutive. Since $j$ is $G$ equivariant, for all $\xi \in \mathfrak{g}$ we have $\left.\xi_{M}\right|_{S}=\xi_{S},\left(j^{*} \mu\right)^{\xi}=j^{*}\left(\mu^{\xi}\right)$, and $\left(j^{*} \alpha\right)^{\xi}=j^{*}\left(\alpha^{\xi}\right)$. Furthermore, by the naturality of the exterior derivative we have

$$
\mathrm{d}\left(j^{*} \mu\right)^{\xi}=\mathrm{d} j^{*}\left(\mu^{\xi}\right)=j^{*}\left(\mathrm{~d} \mu^{\xi}\right),
$$

so $\mathrm{d}\left(\left.\mu\right|_{S}\right)^{\xi}=\left.\left(\mathrm{d} \mu^{\xi}\right)\right|_{S}$. For each $x \in S \subset M$, since $\left.\left(\xi_{M}+\alpha^{\xi}-i \mathrm{~d} \mu^{\xi}\right)\right|_{x} \in E_{x}$, this means that

$$
\left.\left(\xi_{S}+\left.\alpha^{\xi}\right|_{S}-\left.i \mathrm{~d} \mu^{\xi}\right|_{S}\right)\right|_{x} \in E_{S, x}
$$

Again using the $G$-equivariance of $j$, for all $x \in S$ we have

$$
\left.\iota_{\xi_{S}} j^{*}(H)\right|_{x}=\left.j^{*}\left(\iota_{\xi_{S}} H\right)\right|_{x}=\left.j^{*}\left(\iota_{\xi_{M}} H\right)\right|_{x}=\left.j^{*}\left(\mathrm{~d} \alpha^{\xi}\right)\right|_{x}=\left.\mathrm{d}\left(j^{*} \alpha\right)^{\xi}\right|_{x} .
$$

Thus the action of $G$ on $\left(S, E_{S}, j^{*} H\right)$ is twisted Hamiltonian with moment map $\left.\mu\right|_{S}$ and moment one-form $\left.\alpha\right|_{S}$.

The above result holds also for the untwisted case, of course, by putting $H=0$ and $\alpha=0$.

The following three results are exactly what makes reduction of generalized Hamiltonian manifolds possible.

Theorem 4.6 (Lemma 3.8 and Proposition 4.6 of [14]). Let a compact Lie group $G$ act on a $G C$ manifold $(M, \mathcal{J})$, respectfully a $G K$ manifold $\left(M, \mathcal{J}_{1}, \mathcal{J}_{2}\right)$, in a Hamiltonian fashion with moment map $\mu: M \rightarrow \mathfrak{g}^{*}$. Suppose $a \in \mathfrak{g}^{*}$ is an element such that $G$ acts freely on the inverse image $\mu^{-1}\left(\mathcal{O}_{a}\right)$ of the coadjoint orbit $\mathcal{O}_{a}$ of $G$ through a. Then the quotient space $\mu^{-1}\left(\mathcal{O}_{a}\right) / G$ inherits a natural $G C$ structure $\tilde{\mathcal{J}}$ from $\mathcal{J}$, respectfully a natural $G K$ structure $\left(\widetilde{\mathcal{J}}_{1}, \widetilde{\mathcal{J}}_{2}\right)$ from $\left(\mathcal{J}_{1}, \mathcal{J}_{2}\right)$.

Lemma 4.7 (Lemma A.6 of [14]). Let a compact Lie group $G$ act freely on a manifold $M$. Let $H$ be a $G$-invariant and closed three-form, and let $\alpha: \mathfrak{g} \rightarrow \Omega^{1}(M)$ be an equivariant map. Fix a connection $\theta \in \Omega^{1}(M, \mathfrak{g})$ on the principal $G$-bundle $M \rightarrow M / G$. Then if $H+\alpha \in \Omega_{G}^{3}(M)$ is equivariantly closed, there exists a natural form $\Gamma \in \Omega^{2}(M)^{G}$ so that $\iota_{\xi_{M}} \Gamma=\alpha^{\xi}$ for all $\xi \in \mathfrak{g}$. Thus $H+\alpha+\mathrm{d}_{G} \Gamma \in \Omega^{3}(M)^{G} \subset \Omega_{G}^{3}(M)$ is closed and basic and so descends to a closed form $\widetilde{H} \in \Omega^{3}(M / G)$ so that $[\widetilde{H}]=\kappa[H+\alpha]$.

Theorem 4.8 (Propositions A.7 and A.10 of [14]). Let a compact Lie group $G$ act on a twisted $G C$ manifold $(M, \mathcal{J}, H)$, respectfully a twisted $G K$ manifold $\left(M, \mathcal{J}_{1}, \mathcal{J}_{2}, H\right)$, in a Hamiltonian fashion with moment map $\mu: M \rightarrow \mathfrak{g}^{*}$ and moment one-form $\alpha \in \Omega^{1}(M, \mathfrak{g})$. Suppose $a \in \mathfrak{g}^{*}$ is an element such that $G$ acts freely on the inverse image $\mu^{-1}\left(\mathcal{O}_{a}\right)$ of the coadjoint orbit $\mathcal{O}_{a}$ of $G$ through a. Assume that $H+\alpha$ is equivariantly closed. Given a connection on the principal $G$-bundle $\mu^{-1}\left(\mathcal{O}_{a}\right) \rightarrow \mu^{-1}\left(\mathcal{O}_{a}\right) / G$, the quotient space $\mu^{-1}\left(\mathcal{O}_{a}\right) / G$ inherits an $\widetilde{H}$ twisted $G C$ structure $\widetilde{\mathcal{J}}$ from $\mathcal{J}$, respectfully an $\widetilde{H}$-twisted $G K$ structure $\left(\widetilde{\mathcal{J}}_{1}, \widetilde{\mathcal{J}}_{2}\right)$ from $\left(\mathcal{J}_{1}, \mathcal{J}_{2}\right)$, where $\widetilde{H}$ is defined as in Lemma 4.7 above. Up to B-transform, these inherited structures are independent of our choice of connection. 
Definition 4.9. The quotient space $\mu^{-1}\left(\mathcal{O}_{a}\right) / G$ in Theorems 4.6 and 4.8 is called the generalized complex quotient (or generalized Kähler quotient, as applicable), or the LinTolman quotient, of $M$ by $G$ at level $a$. We use the notation

$$
M_{a}:=\mu^{-1}\left(\mathcal{O}_{a}\right) / G
$$

Remark 4.10. As noted in Example 3.9 of [14], in the context of the hypotheses of Theorem 4.6, if the GC structure and moment map come from a symplectic structure and moment map, then the GC structure on the quotient is exactly the one induced by the Marsden-Weinstein ssymplectic structure on the quotient.

The following result will be useful to us later. Its proof follows trivially from the definitions of generalized and twisted generalized Hamiltonian actions.

Lemma 4.11. Let $(M, \mathcal{J}, H)$ be a twisted $G C$ manifold with a Hamiltonian action of a Lie group $G$, moment map $\mu: M \rightarrow \mathfrak{g}^{*}$, and moment one-form $\alpha \in \Omega^{1}\left(M, \mathfrak{g}^{*}\right)$. Let $K \subset G$ be a Lie subgroup. Then the induced action of $K$ on $(M, \mathcal{J}, H)$ is also Hamiltonian, with generalized moment map and moment one-form the compositions of $\mu$ and $\alpha$, respectively, with the projection $\mathfrak{g}^{*} \rightarrow \mathfrak{k}^{*}$ dual to the inclusion $\mathfrak{k} \hookrightarrow \mathfrak{g}$ :

$$
M \stackrel{\mu}{\longrightarrow} \mathfrak{g}^{*} \longrightarrow \mathfrak{k}^{*}, \quad T M \stackrel{\alpha}{\longrightarrow} \mathfrak{g}^{*} \longrightarrow \mathfrak{k}^{*} .
$$

Example 4.12. Let $G$ be a Lie group, and let $\left(M_{i}, \mathcal{J}_{i}, H_{i}\right)$ be a twisted GC manifold on which $G$ acts in a Hamiltonian fashion with moment map $\mu_{i}: M_{i} \rightarrow \mathfrak{g}^{*}$ and moment oneform $\alpha_{i} \in \Omega^{1}\left(M_{i}, \mathfrak{g}^{*}\right)$, for $i=1,2$. Let $\left(M_{1} \times M_{2}, \mathcal{J}, H\right)$ be the product of these two GC manifolds, as defined in Example 2.10. Recall that $\mathcal{J}=\left(\mathcal{J}_{1}, \mathcal{J}_{2}\right)$ and $H=\pi_{1}^{*} H_{1}+\pi_{2}^{*} H_{2}$, where $\pi_{i}: M_{1} \times M_{2} \rightarrow M_{i}$ is the natural projection for $i=1,2$. Define $\mu: M_{1} \times M_{2} \rightarrow \mathfrak{g}^{*} \oplus \mathfrak{g}^{*}$ and $\alpha \in \Omega^{1}\left(M_{1} \times M_{2}, \mathfrak{g}^{*} \oplus \mathfrak{g}^{*}\right)$ by $\mu=\pi_{1}^{*} \mu_{1}+\pi_{2}^{*} \mu_{2}$ and $\alpha=\pi_{1}^{*} \alpha_{1}+\pi_{2}^{*} \alpha_{2}$. It is easy to check that the action of $G \times G$ on $M_{1} \times M_{2}$ is twisted generalized Hamiltonian with moment map $\mu$ and moment one-form $\alpha$.

Embedding $G$ diagonally in $G \times G$, we obtain a Hamiltonian action of $G$ on $M_{1} \times M_{2}$. The projection $\mathfrak{g}^{*} \oplus \mathfrak{g}^{*} \rightarrow \mathfrak{g}^{*}$ induced by this embedding is given by addition: $\left(\lambda_{1}, \lambda_{2}\right) \mapsto \lambda_{1}+\lambda_{2}$, so a moment map and moment one-form for the $G$-action on $M_{1} \times M_{2}$ is given by

$$
M_{1} \times M_{2} \rightarrow \mathfrak{g}^{*}, \quad\left(x_{1}, x_{2}\right) \mapsto \mu_{1}\left(x_{1}\right)+\mu_{2}\left(x_{2}\right)
$$

and

$$
T M_{1} \times T M_{2} \rightarrow \mathfrak{g}^{*}, \quad\left(X_{1}, X_{2}\right) \mapsto \alpha_{1}\left(X_{1}\right)+\alpha_{2}\left(X_{2}\right),
$$

respectively.

Perhaps the most important instance of the construction of Example 4.12 is if we start with an arbitrary twisted generalized Hamiltonian $G$-manifold, $(M, \mathcal{J}, H, \mu, \alpha)$, and let the second GC manifold be a coadjoint orbit $\mathcal{O}_{a}$ in $\mathfrak{g}^{*}$, where $a \in \mathfrak{g}^{*}$ is some fixed element. Let $\omega_{a}$ be the canonical symplectic structure on $\mathcal{O}_{a}$. The action of $G$ on $\mathcal{O}_{a}$ is Hamiltonian in the symplectic sense, with moment map given by the inclusion $\mathcal{O}_{a} \hookrightarrow \mathfrak{g}^{*}$. Using the symplectic structure $-\omega_{a}$ instead, the action is still Hamiltonian, but now the moment map is given by the negative inclusion $\mathcal{O}_{a} \rightarrow \mathfrak{g}^{*}, \lambda \mapsto-\lambda$.

As described in Examples 2.9 and 4.4, the symplectic structure $-\omega_{a}$ induces a GC structure $\mathcal{J}_{a}$ on $\mathcal{O}_{a}$, and the $G$-action on $\mathcal{O}_{a}$ is generalized Hamiltonian with the same moment map. Viewing $\left(\mathcal{O}_{a}, \mathcal{J}_{a}\right)$ as a twisted GC manifold where the twisting is by the zero three-form, the $G$-action 
is twisted generalized Hamiltonian with a constantly vanishing moment one-form. Then the diagonal $G$-action on $M \times \mathcal{O}_{a}$ is twisted generalized Hamiltonian with moment map

$$
\mu^{\prime}: M \times \mathcal{O}_{a} \rightarrow \mathfrak{g}^{*}, \quad(x, \lambda) \mapsto \mu(x)-\lambda
$$

and moment one-form

$$
\alpha^{\prime}: T M \times T\left(\mathcal{O}_{a}\right) \rightarrow \mathfrak{g}^{*}, \quad(X, Y) \mapsto \alpha(X) .
$$

The reason this construction is important is that it is the basis of the shifting trick. If one wishes to reduce $M$ by $G$ at level $a \in \mathfrak{g}^{*}$, one can instead consider the reduction of $M \times \mathcal{O}_{a}$ by $G$ at level 0, because

$$
M_{a} \approx\left(M \times \mathcal{O}_{a}\right)_{0}
$$

as topological spaces. To see this, observe that $\mu^{-1}\left(\mathcal{O}_{a}\right)$ and $\left(\mu^{\prime}\right)^{-1}(0)$ are $G$-equivariantly homeomorphic via the maps

$$
\mu^{-1}\left(\mathcal{O}_{a}\right) \rightarrow\left(\mu^{\prime}\right)^{-1}(0), \quad x \mapsto(x, \mu(x))
$$

and

$$
\left(\mu^{\prime}\right)^{-1}(0) \rightarrow \mu^{-1}\left(\mathcal{O}_{a}\right), \quad(x, \lambda) \mapsto x
$$

\section{Partition of the generalized reduced space}

Let $M$ be a manifold, $G$ be a Lie group acting on $M$ smoothly, and $\mu: M \rightarrow \mathfrak{g}^{*}$ a smooth, $G$ equivariant map. Let $a \in \mathfrak{g}^{*}$. By equivariance the pre-image $\mu^{-1}\left(\mathcal{O}_{a}\right)$ of the coadjoint orbit $\mathcal{O}_{a}$ is preserved by $G$, and so we can consider the quotient space $\mu^{-1}\left(\mathcal{O}_{a}\right) / G$. Let $M=\bigsqcup M_{(H)}$ be the orbit type partition of $M$. Because each set $M_{(H)}$ is stable under $G$, each intersection $\mu^{-1}\left(\mathcal{O}_{a}\right) \cap M_{(H)}$ is also stable under $G$, so the orbit type partition of $M$ descends to a partition

$$
\mu^{-1}\left(\mathcal{O}_{a}\right) / G=\bigsqcup_{(H)}\left(\mu^{-1}\left(\mathcal{O}_{a}\right) \cap M_{(H)}\right) / G
$$

of the quotient $\mu^{-1}\left(\mathcal{O}_{a}\right) / G$.

Suppose now $M$ is a symplectic manifold, the $G$-action is Hamiltonian, and $\mu$ is a moment map. In this case the quotient space $M_{a}:=\mu^{-1}\left(\mathcal{O}_{a}\right) / G$ is called the symplectic reduction, or Marsden-Weinstein quotient, of $M$ at level $a$. The symplectic moment map condition is that $\mathrm{d} \mu^{\xi}=\iota_{\xi_{M}} \omega$ for all $\xi \in \mathfrak{g}$. If $G$ acts freely on $\mu^{-1}\left(\mathcal{O}_{a}\right)$, then each $\xi_{M}$ is nonzero on $\mu^{-1}\left(\mathcal{O}_{a}\right)$, which by the non-degeneracy of $\omega$ implies that $a$ is a regular value of $\mu$. Therefore $\mu^{-1}\left(\mathcal{O}_{a}\right) \subset M$ is a submanifold, so $M_{a}$ is a manifold. In this case, Marsden and Weinstein proved that $M_{a}$ inherits a natural symplectic structure. Theorems 4.6 and 4.8, proved in [14], are analogues of this result.

In the event that the symplectic quotient is singular, one can consider the individual parts of the partitioned quotient. In [19], Lerman and Sjamaar proved that each component of $\left(M_{a}\right)_{(H)}:=\left(\mu^{-1}\left(\mathcal{O}_{a}\right) \cap M_{(H)}\right) / G$ inherits a natural symplectic structure. The main results of this paper are analogues of this in the generalized complex case.

Remark 5.1. By the symplectic moment map condition, $\mathrm{d} \mu^{\xi}=\iota_{\xi_{M}} \omega$, if $a \in \mathfrak{g}^{*}$ is a regular value of $\mu$, then each vector field $\xi_{M}$ is nowhere zero on $\mu^{-1}(a)$. This means that the action of $G$ on $\mu^{-1}(a)$ is at least locally free, which means that the quotient $M_{a}$ is at worst an orbifold, to which Marsden and Weinstein were able to associate a symplectic structure. By Sard's Theorem, 
a generic value of $\mu$ will be regular, so the generic result of symplectic reduction is a symplectic orbifold.

If $(M, \mathcal{J})$ is an untwisted GC manifold with moment map $\mu$, then the generalized moment map condition, $\mathcal{J}\left(\mathrm{d} \mu^{\xi}\right)=-\xi_{M}$, likewise guarantees the equivalence of regular values and local freeness of the action, so $M_{a}$ is at worst an orbifold. However, if $(M, \mathcal{J}, H)$ is a twisted GC manifold with moment map $\mu$ and moment one-form $\alpha$, then this equivalence may no longer hold, due to the presence of the moment one-form in the moment condition:

$$
\mathcal{J}\left(\mathrm{d} \mu^{\xi}\right)=-\xi_{M}-\alpha^{\xi} .
$$

Specifically, $\xi_{M}$ could vanish even if $\mathcal{J}\left(\mathrm{d} \mu^{\xi}\right)$ does not. Therefore, it seems that the generic result of GC reduction may be a GC singular space.

Before stating and proving our main theorem, we need the following lemma.

Lemma 5.2. Let $(M, \mathcal{J}, H)$ be a compact, twisted $G C$ manifold, and let $G$ be a compact Lie group acting on $(M, \mathcal{J}, H)$ in a Hamiltonian fashion with moment map $\mu: M \rightarrow \mathfrak{g}^{*}$ and moment one-form $\alpha \in \Omega^{1}(M, \mathfrak{g})$. If the $G$-action on $M$ is trivial, then $\mathrm{d} \mu=\alpha \equiv 0$.

In Lemma 5.5 of [1], the authors proved the above result in the case that $G$ is a torus; however, their proof holds just as well in the non-abelian case. It relies on viewing the components of $\mu$ as the real parts of a pseudo-holomorphic function and applying a version of the Maximum Principle, a course first taken in [17]. A thorough description of this version of the Maximum Principle can be found in Section 4.4 of [8].

\section{Theorem 5.3 (Singular generalized reduction).}

(a) Let $(M, \mathcal{J})$ be a $G C$ manifold, and let $G$ be a compact group acting in a Hamiltonian fashion on $(M, \mathcal{J})$ with generalized moment map $\mu: M \rightarrow \mathfrak{g}^{*}$. Let $a \in \mathfrak{g}^{*}$, and let $M_{a}=\bigsqcup\left(M_{a}\right)_{(H)}$ be the orbit type partition of the $G C$ quotient of $(M, \mathcal{J})$ by $G$ at level a. Then each component of each $\left(M_{a}\right)_{(H)}$ inherits a natural $G C$ structure from $(M, \mathcal{J})$.

(b) Let $(M, \mathcal{J}, H)$ be a compact $G C$ manifold, and let $G$ be a compact group acting in a Hamiltonian fashion on $(M, \mathcal{J}, H)$ with generalized moment map $\mu: M \rightarrow \mathfrak{g}^{*}$ and moment one-form $\alpha \in \Omega^{1}\left(M, \mathfrak{g}^{*}\right)$. Assume that $H+\alpha$ is equivariantly closed. Let $a \in \mathfrak{g}^{*}$, and $M_{a}=\bigsqcup\left(M_{a}\right)_{(H)}$ be the orbit type partition of the $G C$ quotient of $(M, \mathcal{J}, H)$ by $G$ at level $a$. Then each component of each $\left(M_{a}\right)_{(H)}$ inherits a twisted $G C$ structure from $(M, \mathcal{J}, H)$, natural up to B-transform.

Proof. We begin with the twisted case.

First we prove the theorem in the case that $a=0$.

Let $x \in M$ and $K=G_{x}$. Note that this implies that $K$ is a closed subgroup of $G$, and is hence compact. Clearly $K$ acts canonically on $(M, \mathcal{J}, H)$, since $G$ does. By part (c) of Proposition 3.6, $M_{K}^{l_{x}}$ is open in $M^{K}$. It follows that every component of $M^{K}$ intersecting $M_{K}^{l_{x}}$ has the same dimension as $M_{K}^{l_{x}}$. Let $M_{x}^{K}$ be the union of components of $M^{K}$ having nontrivial intersection with $M_{K}^{l_{x}}$. Since each component of $M^{K}$ is a manifold, it follows that $M_{x}^{K}$ is also. Furthermore, by Proposition 2.19 we know that each connected component of $M^{K}$ is a split submanifold of $(M, \mathcal{J})$, and hence also a twisted GC submanifold. Therefore so is $M_{x}^{K}$.

Let $Z_{K}^{x}$ be the union of components of $M_{K}^{l_{x}}$ that have nontrivial intersection with $\mu^{-1}(0)$. Since $Z_{K}^{x}$ is open in $M_{K}^{l_{x}}$, which is open in $M_{x}^{K}$, as discussed in Remark 2.13 we know that $Z_{K}^{x}$ is a twisted GC submanifold of $M_{x}^{K}$, and hence also of $M$. Let $j: Z_{K}^{x} \hookrightarrow M$ be the inclusion, and denote the $\left(j^{*} H\right)$-twisted GC structure of $Z_{K}^{x}$ by $\mathcal{J}^{\prime}$.

Let $N=N_{G}(K)$ be the normalizer of $K$ in $G$. By part (e) of Proposition 3.6, we know $M_{K}^{l_{x}}$ is $N$-stable. In fact, so is $Z_{K}^{x}$, as we now show. Note that connected components of manifolds 
are path-connected. Therefore, for any $n \in N$, if $y, z \in M_{K}^{l_{x}}$ are in the same component, then so are $n \cdot y$ and $n \cdot z$. Now let $n \in N$ and $y \in Z_{K}^{x}$. By the definition of $Z_{K}^{x}$, there exists some $z$ in the same component of $M_{K}^{l_{x}}$ as $y$ such that $z \in \mu^{-1}(0)$. Since $\mu^{-1}(0)$ is $N$-stable, this means $n \cdot z \in \mu^{-1}(0) \cap M_{K}^{l_{x}} \subset Z_{K}^{x}$, and hence $n \cdot y \in Z_{K}^{x}$ as well.

Now we will show that $\mu\left(Z_{K}^{x}\right)$ and $\alpha\left(T Z_{K}^{x}\right)$ lie in $\operatorname{Ann}_{\mathfrak{g}^{*}}(\mathfrak{k}) \cap\left(\mathfrak{g}^{*}\right)^{K}$, where $\operatorname{Ann}_{\mathfrak{g}^{*}}(\mathfrak{k})$ denotes the annihilator of $\mathfrak{k}$ in $\mathfrak{g}^{*}$. Since $M_{x}^{K}$ is fixed point-wise by $K$ and $\mu$ and $\alpha$ are equivariant, we know these two sets are contained in $\left(\mathfrak{g}^{*}\right)^{K}$. Because $M_{x}^{K}$ is closed in $M$, it is compact. Since $K$ acts trivially on $M_{x}^{K}$, it follows from Theorem 4.5 and Lemmas 4.11 and 5.2 that $\mathrm{d} \mu^{\xi}=\alpha^{\xi}=0$ on $T M_{x}^{K}$, and hence on $T Z_{K}^{x}$, for all $\xi \in \mathfrak{k}$. Hence $\mu^{\xi}$ is locally constant on $Z_{K}^{x}$ for all $\xi \in \mathfrak{k}$. Because each component of $Z_{K}^{x}$ has nonempty intersection with $\mu^{-1}(0)$, it follows that $\mu^{\xi}=0$ on $Z_{K}^{x}$ for all $\xi \in \mathfrak{k}$, so $\mu\left(Z_{K}^{x}\right) \subset \operatorname{Ann}_{\mathfrak{g}^{*}}(\mathfrak{k})$.

Let $L$ denote the quotient Lie group $N / K$, and let $\mathfrak{l}$ denote its Lie algebra. In Lemma 17 of [2], it is proved that the projection $\mathfrak{g}^{*} \rightarrow \mathfrak{n}^{*}$ dual to the inclusion $\mathfrak{n} \hookrightarrow \mathfrak{g}$ induces an isomorphism

$$
\operatorname{Ann}_{\mathfrak{g}^{*}}(\mathfrak{k}) \cap\left(\mathfrak{g}^{*}\right)^{K} \cong \operatorname{Ann}_{\mathfrak{n}^{*}}(\mathfrak{k}) \cong \mathfrak{l}^{*} .
$$

Let $\mu^{\prime}: Z_{K}^{x} \rightarrow \mathfrak{I}^{*}$ and $\alpha^{\prime}: T Z_{K}^{x} \rightarrow \mathfrak{I}^{*}$ be the compositions of this isomorphism with the restrictions of $\mu$ and $\alpha$, respectively, and note that

$$
Z_{K}^{x} \cap \mu^{-1}(0)=Z_{K}^{x} \cap\left(\mu^{\prime}\right)^{-1}(0) .
$$

Because $Z_{K}^{x}$ is fixed point-wise by $K$, the action of $N$ on $Z_{K}^{x}$ induces an action of the quotient $L=N / K$ on $Z_{K}^{x}$. We now verify that this action is twisted generalized Hamiltonian with moment map $\mu^{\prime}$ and moment one-form $\alpha^{\prime}$.

Since $\mu, \alpha$, and the projection $\mathfrak{g}^{*} \rightarrow \mathfrak{n}^{*}$ are $N$-equivariant, and $Z_{K}^{x}$ consists of $K$-fixed points, we know that $\mu^{\prime}$ and $\alpha^{\prime}$ are $L$-equivariant. Now we check that $\mu^{\prime}$ and $\alpha^{\prime}$ satisfy the generalized moment map conditions for the $L$-action on $Z_{K}^{x}$. Because $K$ fixes the points of $Z_{K}^{x}$, the infinitesimal action of $\mathfrak{k}$ on $Z_{K}^{x}$ is zero, so for all $\xi \in \mathfrak{n}$ we have $[\xi]_{Z_{K}^{x}}=\xi_{Z_{K}^{x}}$, where $[\xi]$ denotes the image of $\xi$ under the quotient projection $\mathfrak{n} \rightarrow \mathfrak{n} / \mathfrak{k} \cong \mathfrak{l}$. As noted above, $\mu^{\eta}=0$ and $\alpha^{\eta}=0$ for all $\eta \in \mathfrak{k}$, so $\left(\mu^{\prime}\right)^{[\xi]}=\mu^{\xi}$ and $\left(\alpha^{\prime}\right)^{[\xi]}=\alpha^{\xi}$ for all $\xi \in \mathfrak{n}$. By Theorem 4.5 and Lemma 4.11, the compositions

$$
Z_{K}^{x} \stackrel{\mu}{\longrightarrow} \mathfrak{g}^{*} \longrightarrow \mathfrak{n}^{*} \quad \text { and } \quad T Z_{K}^{x} \stackrel{\alpha}{\longrightarrow} \mathfrak{g}^{*} \longrightarrow \mathfrak{n}^{*}
$$

are a generalized moment map and moment one-form for the $N$-action on the $\left(j^{*} H\right)$-twisted GC manifold $Z_{K}^{x}$, respectively, so we conclude that

$$
[\xi]_{Z_{K}^{x}}=\xi_{Z_{K}^{x}}=-\mathcal{J}^{\prime}\left(\mathrm{d} \mu^{\xi}\right)-\alpha^{\xi}=-\mathcal{J}^{\prime}\left(\mathrm{d}\left(\mu^{\prime}\right)^{[\xi]}\right)-\left(\alpha^{\prime}\right)^{\xi}
$$

and

$$
\iota_{[\xi]_{Z_{K}^{x}}}\left(j^{*} H\right)=\iota_{\xi_{Z_{K}^{x}}}\left(j^{*} H\right)=\mathrm{d} \alpha^{\xi}=\mathrm{d}\left(\alpha^{\prime}\right)^{[\xi]}
$$

for all $[\xi] \in \mathfrak{l}$.

By part (e) of Proposition 3.6, we know that $N / K$ acts freely on $Z_{K}^{x}$, and hence also on $\left(\mu^{\prime}\right)^{-1}(0)$. Since $H+\alpha$ is $G$-equivariantly closed, we know $H$ is closed. Using this fact, our computations from the previous paragraph, and part (b) of Remark 4.3, we compute

$$
\begin{aligned}
\mathrm{d}_{L}\left(j^{*} H+\alpha^{\prime}\right)([\xi]) & =\mathrm{d}\left(j^{*} H\right)-\iota_{[\xi]_{Z_{K}^{x}}}\left(j^{*} H\right)+\mathrm{d}\left(\alpha^{\prime}\right)^{[\xi]}-\iota_{[\xi]_{Z_{K}^{x}}}\left(\alpha^{\prime}\right)^{[\xi]} \\
& =j^{*}(\mathrm{~d} H)-\iota_{\xi_{Z_{K}^{x}}}\left(j^{*} H\right)+\mathrm{d} \alpha^{\xi}-\iota_{\xi_{Z_{K}^{x}}}\left(\alpha^{\xi}\right) \\
& =0-\mathrm{d} \alpha^{\xi}+\mathrm{d} \alpha^{\xi}-0=0
\end{aligned}
$$


for all $[\xi] \in(\mathfrak{n} / \mathfrak{k})^{*} \cong \mathfrak{l}^{*}$. Hence $j^{*} H+\alpha^{\prime}$ is $L$-equivariantly closed. Therefore we can apply Lin-Tolman's twisted generalized reduction, Theorem 4.8 above, and obtain a GC structure on the quotient space

$$
\left(\mu^{\prime}\right)^{-1}(0) /(N / K) \cong\left(\mu^{\prime}\right)^{-1}(0) / N \cong\left(Z_{K}^{x} \cap \mu^{-1}(0)\right) / N
$$

Recall that this structure is only natural up to $B$-transform. It follows that each component of $\left(Z_{K}^{x} \cap \mu^{-1}(0)\right) / N$ is a twisted GC manifold.

By varying the point $x \in M_{K}$, and thus varying the manifold $Z_{K}^{x}$, we can conclude that every component $\left(M_{K} \cap \mu^{-1}(0)\right) / N$ is a twisted GC manifold.

By parts (d) and (f) of Proposition 3.6, we know that $G \cdot M_{K}=M_{(K)}$ and that the inclusion $M_{K} \hookrightarrow M_{(K)}$ induces a homeomorphism $M_{K} / N \approx M_{(K)} / G$. Together with the fact that $\mu^{-1}(0)$ is $G$-stable, this first fact implies that $G \cdot\left(M_{K} \cap \mu^{-1}(0)\right)=M_{(K)} \cap \mu^{-1}(0)$. Together with the second fact, this implies that

$$
\left(M_{0}\right)_{(K)}:=\left(M_{(K)} \cap \mu^{-1}(0)\right) / G \approx\left(M_{K} \cap \mu^{-1}(0)\right) / N,
$$

and so each component of $\left(M_{0}\right)_{(K)}$ inherits a twisted GC structure, natural up to $B$-transform.

The general case, where the reduction is taken at an arbitrary level $a \in \mathfrak{g}^{*}$ now follows from the shifting trick, as explained following Example 4.12 above.

Now we consider the untwisted case. Since an untwisted Hamiltonian GC manifold is simply a twisted Hamiltonian GC manifold with $H=0$ and $\alpha=0$, the only real difference between parts (a) and (b) of this theorem is that in part (a) we do not assume that $M$ is compact. Note that the only time above where we used the fact that $M$ is compact was when showing that $\mu^{\xi}$ and $\alpha^{\xi}$ both vanish on $Z_{K}^{x}$ for all $\xi \in \mathfrak{k}$, and hence that $\mu\left(Z_{K}^{x}\right)$ and $\alpha\left(T Z_{K}^{x}\right)$ lie in $\operatorname{Ann}_{\mathfrak{g}^{*}}(\mathfrak{k})$. For this non-compact case, note that since $Z_{K}^{x}$ contains only $K$-fixed points, we have $\xi_{Z_{K}^{x}}=0$ for all $\xi \in \mathfrak{k}$, so

$$
\mathrm{d} \mu^{\xi}=\mathcal{J}^{\prime}\left(\xi_{Z_{K}^{x}}\right)=\mathcal{J}^{\prime}(0)=0
$$

and hence $\mu^{\xi}$ is locally constant on $Z_{K}^{x}$ for all $\xi \in \mathfrak{k}$. Because each component of $Z_{K}^{x}$ has nonempty intersection with $\mu^{-1}(0)$, it follows that $\mu^{\xi}=0$ for all $\xi \in \mathfrak{k}$, so $\mu\left(Z_{K}^{x}\right) \subset \operatorname{Ann}_{\mathfrak{g}^{*}}(\mathfrak{k})$. This completes the proof of (a).

Corollary 5.4 (Singular generalized Kähler reduction). The results of Theorem 5.3 hold if all $G C$ and twisted GC structures are replaced by GK and twisted GK structures, respectively.

Proof. Suppose $\left(M, \mathcal{J}_{1}, \mathcal{J}_{2}\right)$ is a GK manifold, twisted or untwisted. Because a generalized Hamiltonian action on the GK manifold $\left(M, \mathcal{J}_{1}, \mathcal{J}_{2}\right)$ is simply a generalized Hamiltonian action on the GC manifold $\left(M, \mathcal{J}_{1}\right)$ which also preserves the structure $\mathcal{J}_{2}$, it is easy to check that the proof of Theorem 5.3 holds in precisely the same way for our present situation. We will simply note that, for any Lie subgroup $K$ of $G$, because both $\mathcal{J}_{1}$ and $\mathcal{J}_{2}$ are preserved by $K$, by Proposition 2.19 we know that each component of $M^{K}$ is a split submanifold of $M$ with respect to both GC structures, so it is a GK manifold. Everything else is entirely straightforward to check.

\section{Acknowledgements}

The author would like to thank Reyer Sjamaar for his help in understanding singular reduction in the symplectic case, Yi Lin for several extremely helpful conversations, Tomoo Matsumara for introducing him to generalized complex geometry, the referees for many useful suggestions, and his family and friends for their unwavering support. 


\section{References}

[1] Baird T., Lin Y., Topology of generalized complex quotients, J. Geom. Phys. 60 (2010), 1539-1557, arXiv:0802.1341.

[2] Bates L., Lerman E., Proper group actions and symplectic stratified spaces, Pacific J. Math. 181 (1997), 201-229, dg-ga/9407003.

[3] Ben-Bassat O., Boyarchenko M., Submanifolds of generalized complex manifolds, J. Symplectic Geom. 2 (2004), 309-355, math.DG/0309013.

[4] Bursztyn H., Cavalcanti G.R., Gualtieri M., Reduction of Courant algebroids and generalized complex structures, Adv. Math. 211 (2007), 726-765, math.DG/0509640.

[5] Courant T.J., Dirac manifolds, Trans. Amer. Math. Soc. 319 (1990), 631-661.

[6] Cushman R., Śniatycki J., Differential structure of orbit spaces, Canad. J. Math. 53 (2001), 715-755.

[7] Duistermaat J.J., Kolk J.A.C., Lie groups, Universitext, Springer-Verlag, Berlin, 2000.

[8] Goldberg T.E., Hamiltonian actions in integral Kähler and generalized complex geometry, PhD Thesis, Cornell University, 2010.

[9] Gualtieri M., Generalized complex structures, PhD Thesis, University of Oxford, 2003, math.DG/0401221.

[10] Guillemin V.W., Sternberg S., Supersymmetry and equivariant de Rham theory, Mathematics Past and Present, Springer-Verlag, Berlin, 1999.

[11] Hitchin N., Generalized Calabi-Yau manifolds, Q. J. Math. 54 (2003), 281-308, math.DG/0209099.

[12] Hu S., Hamiltonian symmetries and reduction in generalized geometry, Houston J. Math. 35 (2009), 787-811, math.DG/0509060.

[13] Jotz M., Ratiu T.S., Śniatycki J., Singular reduction of Dirac structures, arXiv:0901.3062.

[14] Lin Y., Tolman S., Symmetries in generalized Kähler geometry, Comm. Math. Phys. 268 (2006), 199-222, math.DG/0509069.

[15] Marsden J., Weinstein A., Reduction of symplectic manifolds with symmetry, Rep. Math. Phys. 5 (1974), 121-130.

[16] Meyer K.R., Symmetries and integrals in mechanics, in Dynamical Systems (Proc. Sympos., Univ. Bahia, Salvador, 1971), Academic Press, New York, 1973, 259-272.

[17] Nitta Y., Convexity properties of generalized moment maps, J. Math. Soc. Japan 61 (2009), 1171-1204, arXiv:0901.0361.

[18] Ortega J.-P., Ratiu T.S., Momentum maps and Hamiltonian reduction, Progress in Mathematics, Vol. 222, Birkhäuser Boston, Inc., Boston, MA, 2004.

[19] Sjamaar R., Lerman E., Stratified symplectic spaces and reduction, Ann. of Math. (2) 134 (1991), 375-422.

[20] Stiénon M., Xu P., Reduction of generalized complex structures, J. Geom. Phys. 58 (2008), 105-121, math.DG/0509393. 